\title{
A Mathematical Model for Viscosity in Capillary Extrusion of Two-Component Polyblends*
}

\author{
Chen-Chong LIN \\ Department of Chemical Engineering, National Taiwan \\ University, Taipei, Taiwan, China.
}

(Received February 27, 1978)

\begin{abstract}
Experimental viscosity of two component polyblends is correlated to a mathematical model by introducing a frictional correction factor at the interfaces between two unlike adjacent compositions. It is found that the phase structure as well as the elastic behavior of the compositions can greatly affect the viscosity of the system and make the estimation more complicated. Fair agreement between theory and experiment is found, however, for less compatible systems, such as the PP/PS and PE/PS systems. A lesser degree of acceptance is shown for systems containing an elastic component, such as PVC/ABS and PS/PB systems. The confused phase structure of the systems as evaluated by the electron microscopic observation often causes an increase in viscosity of the systems and thus a deviation from the theoretical calculation.
\end{abstract}

KEY WORDS Viscosity / Two-Component Polyblends / Frictional Correction Factor / Phase Structure / Elastic Behavior /

In recent years, an increasing number of commercial products have appeared on the market, made of more than one polymeric material. Consequently considerable interest has been aroused toward understanding the fundamental nature of the flow properties of polymer blend systems. Useful mixtures were developed, however, in an empirical manner, largely due to the lack of suitable models to represent the effect of the composition on their viscosity. A composition triangle versus property is a convenient means for determining the effect of the composition on the properties of tri-component polymer blends by means of a simple lattice planning of the experiment. ${ }^{1}$ But, parameters obtained were quite empirical and had few physical meanings. Much experimental data on the viscosity of two-component polyblends has appeared in the past years. $^{2,3,4}$ The development of a single mathematical model for predicting the flow behavior of all such polyblends has not been successful. A

* Read at The Second Pacific Chemical Engineering Congress (Pache '77), Denver, Collorado, on August 28 (1977). model of the laminar flow in a pipe of concentric adjacent fluids with the different viscosity was first proposed by Heitmiller ${ }^{5}$ to try to explain the flow viscosity of a polymer-wax blend. The experimental viscosity of two-component polyblends in this investigation, is now correlated to a mathematical model by introducing a frictional correction factor at the liquid interfaces between two unlike adjacent compositions.

\section{THEORETICAL CONSIDERATION}

The author proposes herewith a model of the laminar flow in a pipe of two concentric unlike adjacent fluids having different viscosity but the same density as an approximation of two-component systems to evaluate the relationship between the composition and the viscosity of the mixture. Though the model proposed here is far from the phase structure of the polyblends, from a morphological point of view, the liquid interface will suffice to indicate approximately the entire contact area between the two unlike adjacent compositions [see Appendix]. The derivation of the mass flow rate for the system of fluids follows 

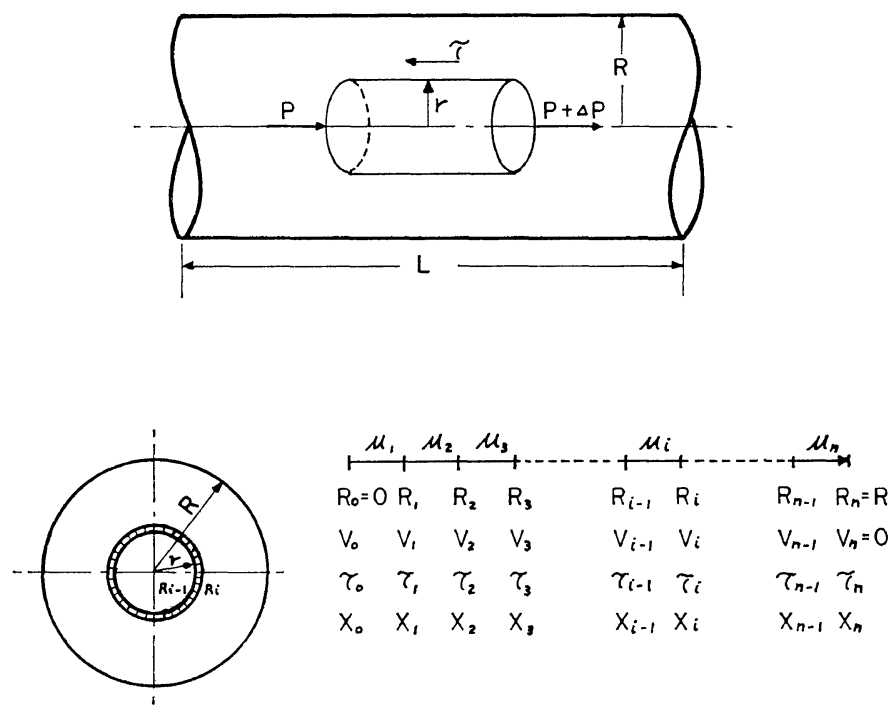

Figure 1. Schematic representation of symbols used.

the same lines as the derivation of the Poiseuille equation.

A sketch illustrating the symbols used in this model is shown in Figure 1. The boundary conditions chosen in our derivations were zerovelocity at the wall, and continuity of alternate shearing stress and friction between the two components at the liquid interfaces.

Under the circumstances, the balance of forces on a cylinder of $r$ where $R_{i-1}<r<R_{i}$ and a frictional correction factor $X$ on the model, lead to the following equation.

$$
\tau=-\mu_{i} \frac{\mathrm{d} v}{\mathrm{~d} r}+X=\frac{-\Delta P}{2 L} r
$$

where $X$ deals with the difference in frictional force between pure-single and two-component polymeric systems. Thus $X$ can be regarded as the frictional force between interfaces of the two unlike adjacent polymeric components and is, therefore, a function of $r$ and $\omega$ at a given temperature and shear rate. Then we have

$$
-\mathrm{d} v=\left(\frac{-\Delta P}{2 L \mu_{i}}\right) r \mathrm{~d} r-\frac{X}{\mu_{i}} \mathrm{~d} r
$$

Integration of eq 2 between the limits $r$ and $R_{i}$ gives the velocity profile in the $i$ th layer, $i . e$., $R_{i-1}<r<R_{i}$

$$
v-V_{i}=\frac{-(\Delta P+2(L / R) Z)}{4 L \mu_{i}}\left(R_{i}{ }^{2}-r^{2}\right)
$$

where $X=r Z / R$ and $Z$ is now a function of $\omega$ only.

The velocity in the interface $r=R_{i-1}$ is

$$
V_{i-1}-V_{i}=\frac{-(\Delta P+2(L / R) Z)}{4 L \mu_{j}}\left({R_{i}}^{2}-R_{j-1}^{2}\right)
$$

The value of $V_{i}$ thus can be evaluated using iterative procedures, starting from layer $n$ (where $r=R, V_{n}=0$ ).

$$
V_{i}=\frac{-(\Delta P+2(L / R) Z)}{4 L} \sum_{j=i+1}^{j=n} \frac{1}{\mu_{j}}\left(R_{j}{ }^{2}-R_{j-1}^{2}\right)
$$

and the velocity profile in layer $i$ is

$$
\begin{aligned}
v= & \frac{-(\Delta P+2(L / R) Z)}{4 L} \sum_{j=i+1}^{j=1} \frac{1}{\mu_{j}}\left(R_{j}{ }^{2}-R_{j-1}^{2}\right) \\
& +\frac{-(\Delta P+2(L / R) Z)}{4 L \mu_{i}}\left({R_{i}}^{2}-r^{2}\right)
\end{aligned}
$$

The volume flow rate in layer $i$ is

$$
\begin{aligned}
Q_{i}= & \int_{R_{i-1}}^{R_{i}} 2 \pi v r \mathrm{~d} r=2 \pi \int_{R_{i-1}}^{R_{i}} \frac{-(\Delta P+2(L / R) Z)}{4 L} \\
& \times \sum_{j=i+1}^{j=n} \frac{1}{\mu_{j}}\left(R_{j}{ }^{2}-R_{j-1}{ }^{2}\right) r \mathrm{~d} r \\
& +2 \pi \int_{R_{i-1}}^{R_{i}} \frac{-(\Delta P+2(L / R) Z)}{4 L \mu_{i}}\left(R_{i}{ }^{2} r-r^{3}\right) \mathrm{d} r \\
= & \frac{-(\Delta P+2(L / R) Z)\left(R_{i}{ }^{2}-R_{i-1}{ }^{2}\right)}{4 L}
\end{aligned}
$$

Polymer J., Vol. 11, No. 3, 1979 


$$
\begin{aligned}
& \times \sum_{j=i+1}^{n} \frac{1}{\mu_{j}}\left(R_{j}{ }^{2}-R_{j-1}^{2}\right) \\
& +\frac{-\pi(\Delta P+2(L / R) Z)\left(R_{i}{ }^{2}-R_{i-1}^{2}\right)^{2}}{8 L \mu_{i}}
\end{aligned}
$$

The total volume flow rate, $Q_{\mathrm{T}}$, then is

$$
Q_{\mathrm{T}}=\sum_{i=1}^{n} Q_{i}
$$

Carrying out the integration and evaluating the summations we have finally

$$
\begin{aligned}
Q_{\mathrm{T}}= & \frac{-\pi(\Delta P+2(L / R) Z)}{8 L} \sum_{i=1}^{n} \frac{1}{\mu_{i}}\left(R_{i}{ }^{2} R_{i-1}{ }^{2}-R_{i-1}^{4}\right) \\
& +\frac{-\pi(\Delta P+2(L / R) Z)}{8 L} \sum_{i=1}^{n} \frac{\left(R_{i}{ }^{2}-R_{i-1}\right)^{2}}{\mu_{i}} \\
& =\frac{-\pi(\Delta P+2(L / R) Z)}{8 L} \sum_{i=1}^{n} \frac{1}{\mu_{i}}\left(R_{i}{ }^{4}-R_{i-1}^{4}\right)
\end{aligned}
$$

From the macroscopic point of view, we know for a fluid of the same density of viscosity $\mu_{\mathrm{E}}$ that

$$
Q_{\mathrm{E}}=\frac{\pi(-\Delta P)}{8 L \mu_{\mathrm{E}}} R^{4}
$$

Setting $Q_{E}=Q_{T}$, we can solve for the effective viscosity $\mu_{\mathrm{E}}$ :

$$
\begin{aligned}
\frac{R^{4}}{\mu_{\mathrm{E}}} & =\frac{\Delta P+2(L / R) Z}{\Delta P} \sum_{i=1}^{n} \frac{R_{i}{ }^{4}-R_{i-1}^{4}}{\mu_{i}} \\
& =\beta \sum_{i=1}^{n} \frac{R_{i}{ }^{4}-R_{i^{-1}}}{\mu_{i}}
\end{aligned}
$$

where

$$
\begin{aligned}
\beta & =\frac{\Delta P+2(L / R) Z}{\Delta P}=1+2\left(\frac{L}{R}\right)\left(\begin{array}{c}
Z \\
\Delta P
\end{array}\right) \\
& =1+4\left(\frac{L}{D}\right)\left(\frac{Z}{\Delta P}\right)
\end{aligned}
$$

Since

$$
\tau_{\mathrm{w}}=\frac{D(-\Delta P)}{4 L}
$$

where $\tau_{\mathrm{w}}$ is the shearing stress at the tube wall. Thus we get

$$
\beta=1-\frac{Z}{\tau_{\mathrm{w}}}
$$

Here, we set $Z$ to be

$$
Z=\alpha_{\mathrm{AB}} \sqrt{\omega_{\mathrm{\Lambda}} \omega_{\mathrm{B}}}
$$

where $\alpha_{\mathrm{AB}}$ represents the interaction coefficient, a constant for a given system. The general case was particularized for a system of two different fluids $A$ and $B$ each occupying in an alternating manner $n / 2$ layers. The weight or volume fractions of the fluids were assumed to be variable, i. e., $\omega_{\mathrm{A}}$ for $A,\left(1-\omega_{\mathrm{A}}\right)$ or $\omega_{\mathrm{B}}$ for $B$, but it was assumed that all annuli of a given fluid had the same area. Since for most polymer melts, which exhibit almost the same density, for the tube of radius $R, A$ occupies $n / 2$ layers, each of area $2 \pi \omega_{\Delta}$ $R^{2} / n$, and $B$ occupies $n / 2$ layers, each of area $2 \pi \omega_{B}$ $R^{2} / n$.

By using eq 9, the two components, $A$ and $B$, are separated, and the summation sign expanded.

$$
\frac{R^{4}}{\mu_{\mathrm{E}}}=\beta\left[\sum_{i=1}^{n / 2} \frac{R_{2 i-1}^{4}-R_{2 i-2}^{4}}{\mu_{\mathrm{A}}}+\sum_{i=1}^{n / 2} \frac{R_{2 i}^{4}-R_{2 i-1}^{4}}{\mu_{\mathrm{B}}}\right]
$$

For component $A$, this gives

$$
\begin{aligned}
\sum_{i=1}^{n / 2}\left(R_{2 i-1}^{4}-R_{2 i-2}^{4}\right) & =\frac{2 \omega_{\Delta} R^{2}}{n} \sum_{i=1}^{n / 2}\left(R_{2 i-1}^{2}+R_{2 i-2}^{2}\right) \\
& =\frac{2 \omega_{\Delta} R^{2}}{n} \frac{R^{2}\left(n-2+2 \omega_{\Delta}\right)}{2} \\
& =\frac{\omega_{\Delta} R^{4}\left(n-2+2 \omega_{\Delta}\right)}{n}
\end{aligned}
$$

For component $B$ under the same procedure,

$$
\sum_{i=1}^{n / 2}\left(R_{2 i}^{4}-R_{2 i-1}^{4}\right)=\frac{\omega_{\mathrm{B}} R^{4}\left(n+2 \omega_{\mathrm{A}}\right)}{n}
$$

The following expression for $\mu_{\mathrm{E}}$ is obtained as

$$
\mu_{\mathrm{E}}=\beta^{-1}\left[\frac{1}{\frac{\omega_{\mathrm{A}}\left(n-2+2 \omega_{\mathrm{A}}\right)}{n \mu_{\mathrm{A}}}+\frac{\omega_{\mathrm{B}}\left(n+2 \omega_{\mathrm{A}}\right)}{n \mu_{\mathrm{B}}}}\right]
$$

If $n \gg 1$, [see Appendix] then,

$$
\mu_{\mathrm{E}}=\beta^{-1} \frac{1}{\frac{\omega_{\mathrm{A}}}{\mu_{\mathrm{A}}}+\frac{\omega_{\mathrm{B}}}{\mu_{\mathrm{B}}}}
$$

or

$$
\frac{1}{\mu_{\mathrm{E}}}=\beta\left[\frac{\omega_{\mathrm{A}}}{\mu_{\mathrm{A}}}+\frac{\omega_{\mathrm{B}}}{\mu_{\mathrm{B}}}\right]
$$

Equation 17 gives the effective viscosity of a system of two-component polyblends. If $\beta=1$, namely $Z=0$, eq 17 coincides with the equation of Heitmiller $^{5}$ as follows: 


$$
\frac{1}{\mu_{\mathrm{E}}}=\frac{\omega_{\mathrm{A}}}{\mu_{\mathrm{A}}}+\frac{\omega_{\mathrm{B}}}{\mu_{\mathrm{B}}}
$$

If because of sufficiently less friction between like components than between unlike components, the values of $\beta$ for most of the polymer mixtures in a given system are larger than unity, then the effective shearing action will be reduced and, therefore, one obtains a lower viscosity than would be obtained by eq 18 .

\section{EXPERIMENTAL}

Systems of PVC/ABS and PS/PB studied were prepared in this paper. The experimental data for another two systems of PE/PS and PP/PS were adapted from data by $\mathrm{Han}$ and $\mathrm{Yu} .^{2,3}$ The value of $\tau_{\mathrm{W}}$ was experimentally obtained from shear stress vs. shear rate plot for a given system. A capillary extruding type of Koka-flow tester was used for obtaining the flow curves. The melt blending was accomplished by an internal mixer (Plasti-Corder, Brabender).

\section{RESULTS AND DISCUSSION}

The value of the correction factor $X$ can be calculated readily from the relation $\tau=\mu \dot{\gamma}+X$, where $\dot{\gamma}$ is shear rate. The experimental viscosity of the pure component may be cited in the above equation to give $X=0$. For the polyblend systems, the values of $\mu$ obtained from eq 18 are substituted into the above equation yielding the values of $X$ for different blend ratios. The solid

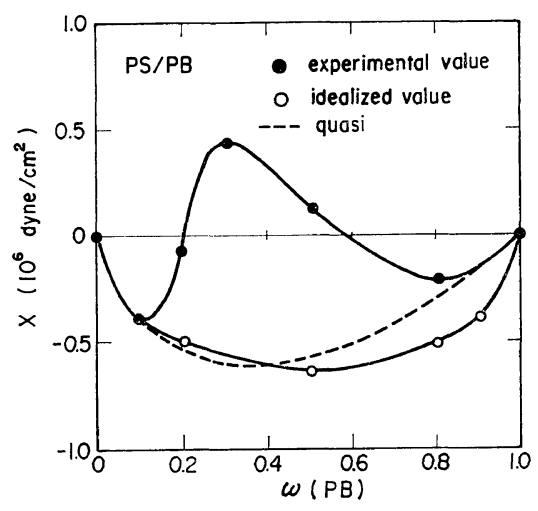

Figure 2. The correction factor of the PS/PB blends as a function of the weight fraction of PB.

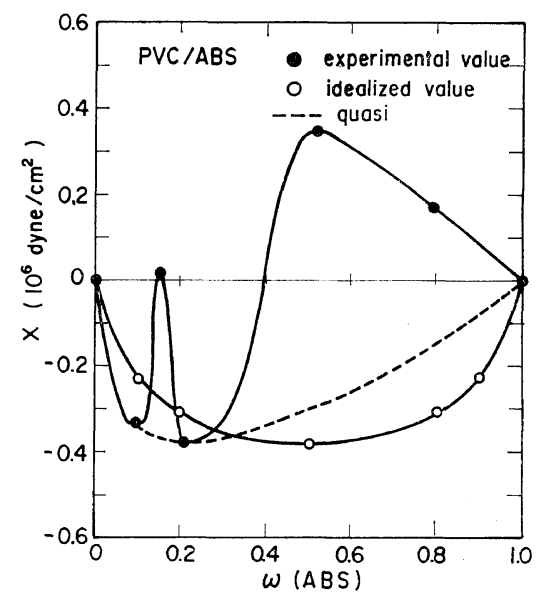

Figure 3. The correction factor of the PVC/ABS blends as a function of the weight fraction of ABS.

Table I. Interaction coefficients evaluated

\begin{tabular}{|c|c|c|c|c|}
\hline System & $\mu_{\mathrm{A}}$, poise & $\mu_{\mathrm{B}}$, poise & $-\alpha, \mathrm{dyn} / \mathrm{cm}^{2}$ & Remark \\
\hline $\mathrm{PS} / \mathrm{PB}$ & $4.4 \times 10^{4}$ & $4.0 \times 10^{4}$ & $1.28 \times 10^{6}$ & $\begin{array}{l}T=145^{\circ} \mathrm{C} \\
D_{\mathrm{W}}=60 \mathrm{~s}^{-1} \\
L / R=20\end{array}$ \\
\hline PVC/ABS & $2.5 \times 10^{4}$ & $5.8 \times 10^{4}$ & $7.67 \times 10^{5}$ & $\begin{array}{l}T=180^{\circ} \mathrm{C} \\
D_{\mathrm{W}}=60 \mathrm{~s}^{-1} \\
L / R=20\end{array}$ \\
\hline $\mathrm{PP} / \mathrm{PS}$ & $2.7 \times 10^{3 \mathrm{a}}$ & $3.1 \times 10^{3 \mathrm{a}}$ & $4.31 \times 10^{5}$ & $\begin{array}{l}T=200^{\circ} \mathrm{C} \\
D_{\mathrm{W}}=200 \mathrm{~s}^{-1} \\
L / R=40\end{array}$ \\
\hline PE/PS & $4.23 \times 10^{3 \mathrm{a}}$ & $3.10 \times 10^{3 a}$ & $3.65 \times 10^{5}$ & $\begin{array}{l}T=200^{\circ} \mathrm{C} \\
D_{\mathrm{W}}=200 \mathrm{~s}^{-1} \\
L / R=40\end{array}$ \\
\hline
\end{tabular}

a Extracted from the data by $\mathrm{Han}$ and $\mathrm{Yu}^{2,3}$ 
lines on Figures 2 to 5 correspond to the experimental values of $X$ as a function of the blending ratio. An abruption is observed at the composition of $\mathrm{PVC} / \mathrm{ABS}=85 / 15$. For convenience, a quasi curve of $X$, shown as the dashed line, is drawn in order to estimate the value of the interaction coefficient, $\alpha$. This average value of $\alpha$ can then be taken to yield the idealized curves of $X$ as shown by using eq 13 . The evaluated mean values for the four polyblend systems are given in Table I. Therefore, the comparisons of the experimental and quasi values of $X$ for each polyblend system are shown in Figures 2 to 5 .

The value of $\beta$ can be calculated from eq 12 as long as the idealized value of $Z$ is available. The theoretical viscosity may then be obtained

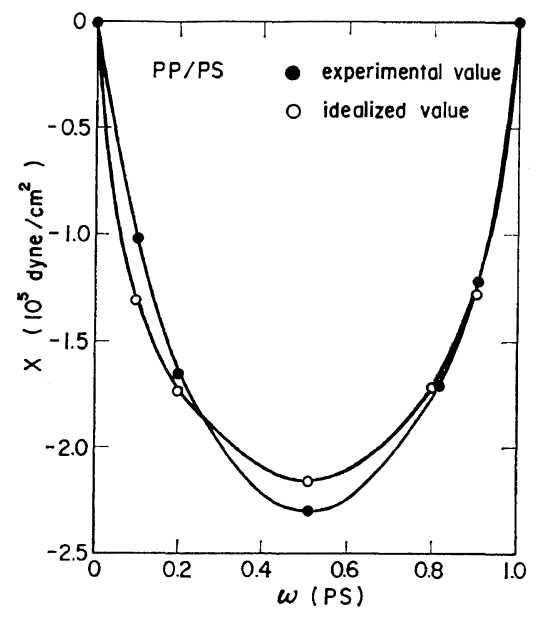

Figure 4. The correction factor of the PP/PS blends as a function of the weight fraction of PS.

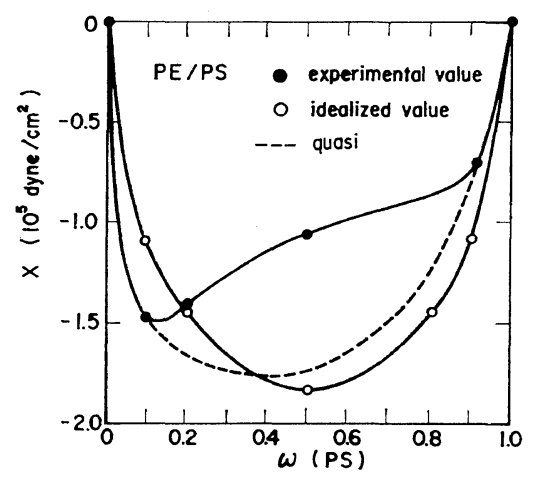

Figure 5. The correction factor of the PE/PS blends as a function of the weight fraction of PS. according to eq 17.

The comparisons of the experimental and theoretical $v s$. viscosity blending ratio curves for such systems are shown in Figures 6, 7, 8, and 9, respectively.

It may be seen that the curves calculated from eq 17 give generally better representations of the experimental data than those calculated from eq 18. The calculated data in accordance with eq 17 for PP/PS system coincides best with the experimental data. It was observed in the PVC/ABS system that the composition at $\mathrm{PVC} /$ $\mathrm{ABS}=85 / 15$ shows abrupt peaks in the experimental melt viscosity. In the PS/PB system, however, a deviation from the experimental data over broad compositions was observed. The compositions of $\mathrm{PB}$ and ABS behave more elastically. While the resin can flow when it melts, the rubbery phase is probably only deformable and does not flow in the ordinary sense. In the PE/PS system, the deviation occurs also at the blending ratio of $\mathrm{PE} / \mathrm{PS}=50 / 50$. These deviations may be attributed, therefore, to the phase fracture as well as the elastic behavior of the component. It is well known that the state of dispersion in a blended system affects the melt viscosity of the system. ${ }^{4}$ At the phase fracture, the assumption of a laminar flow in a pipe is no longer valid. It is easy to be felt that the state of the confused phase structure is in a greater degree of chain entanglement and consequently the effective viscosity of the system increases. The feature of the phase fracture in a certain blending ratio can be grasped with the aid of microphotographs as shown in Figure 6 for PS/PB system. It is also well known that the phase structure of a polyblend can be affected by both the mixing torque ratio and the composition. ${ }^{6}$

The dispersed particles in the suspending medium show a finer state in the composition below $10 \%$ of $\mathrm{PB}$, where $\mathrm{PB}$ consist of dispersed particles. Within these ranges, the experimental viscosity fits well with eq 17 . In the range over $10 \%$ of PB the state of dispersed particles is obscure and the phase structure is more confused. It should be recognized that the effective viscosity under this condition gives a greater value than that calculated, as seen in Figure 7 for the PVC/ABS= $85 / 15$. This may result in an abrupt peak in viscosity. At this instance, no distinct dispersed 


\section{C.-C. LIN}

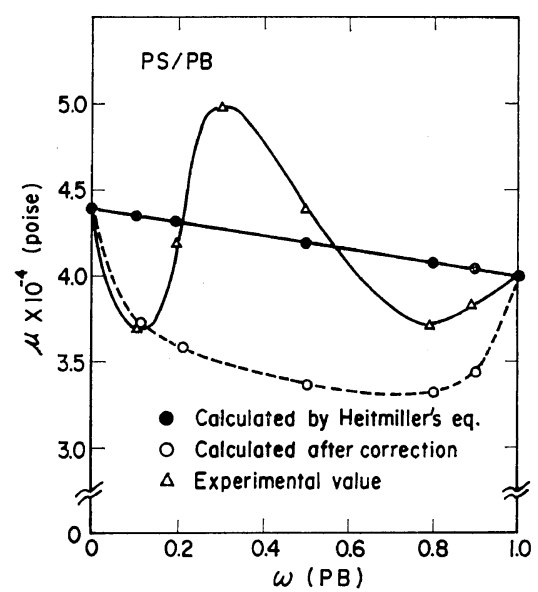

Figure 6. Effect of blending ratio on the melt viscosities of PS/PB blends.

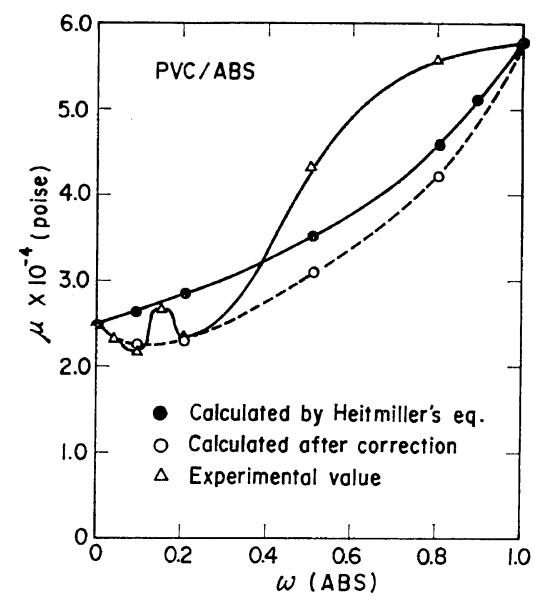

Figure 7. Effect of blending ratio on the melt viscosities of PVC/ABS blends.

particles could be observed by phase micrographs due to the compatibility of these two components, and a poor agreement between experimental and theoretical viscosities resulted. The actual situation is, however, not well understood from a molecular point of view, and it is far more complicated than the simple graphical discussion presented here.

\section{CONCLUSION}

Although the interpretation of the melt viscosity of polyblends is not easy, the results of the fore-

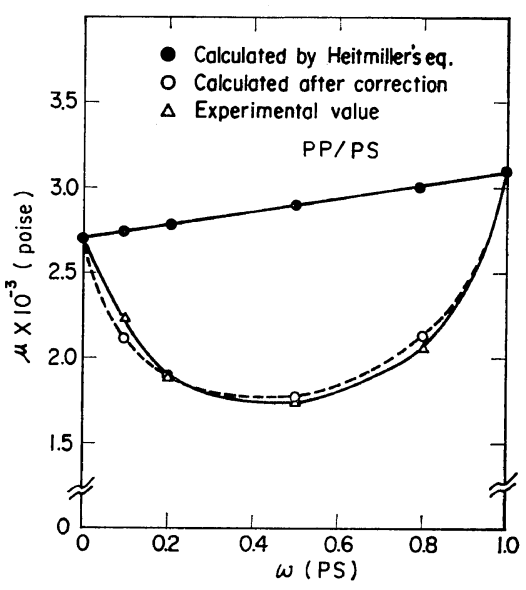

Figure 8. Effect of blending ratio on the melt viscosities of PP/PS blends.

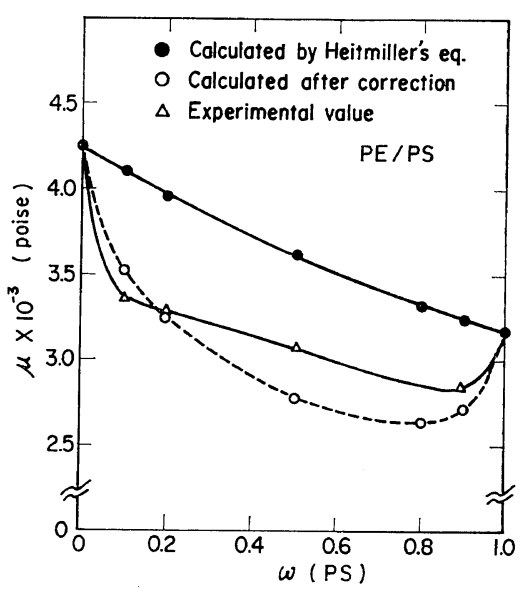

Figure 9. Effect of blending ratio on the melt viscosities of PE/PS blends.

going theory are rather promising. It was noticed above that the phase structure as well as elastic behavior can greatly affect the viscosity of the system and make the estimation of viscosity more complicated. Therefore, one can not grasp the true flow behavior unless there is an understanding of both the morphology and the viscoelastic behavior of the system. However, the flow behavior may be included in the following criteria:

(1) Before the frictional force between two adjacent unlike fluids is applied, the agreement between theory and experimental is generally poor. Better agreement is now found with the proposed 


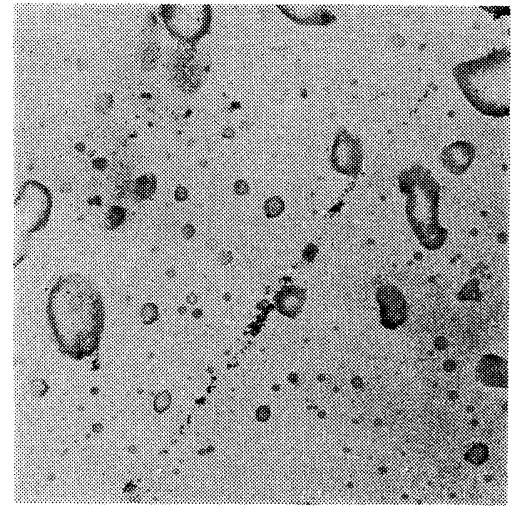

(a)

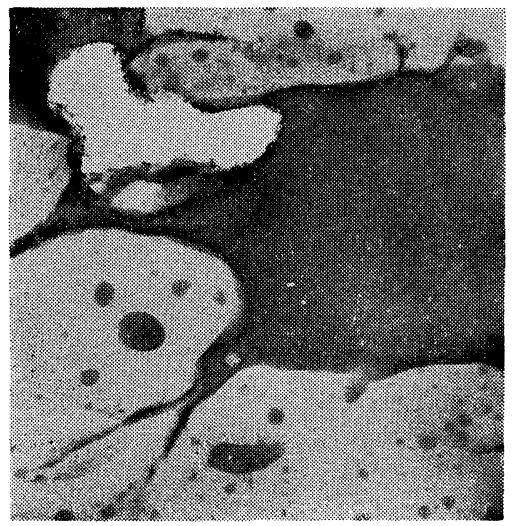

(c)

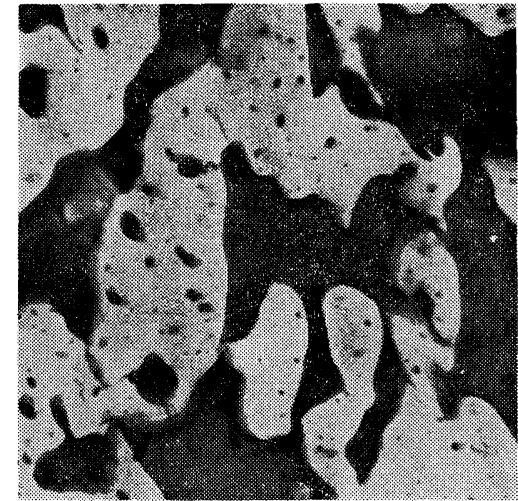

(b)

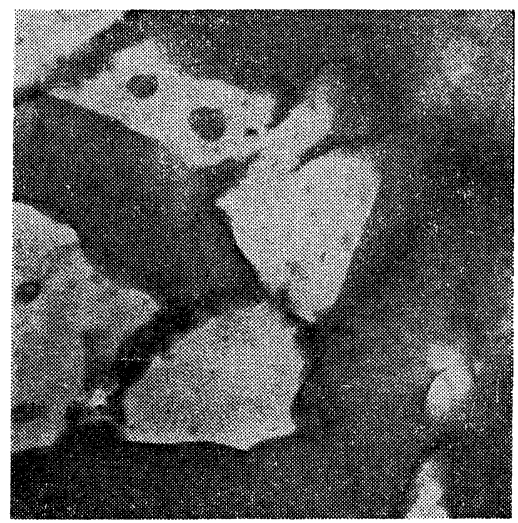

(d)

Figure 10. Electron micrograph $(\times 3,000)$ of the extrudate cross section for $\mathrm{PS} / \mathrm{PB}$ blends: (a), $\mathrm{PS} / \mathrm{PB}=90 / 10$; (b), 70/30; (c), 50/50; (d), 20/80; stained and hardened by the osmium tetroxide procedure.

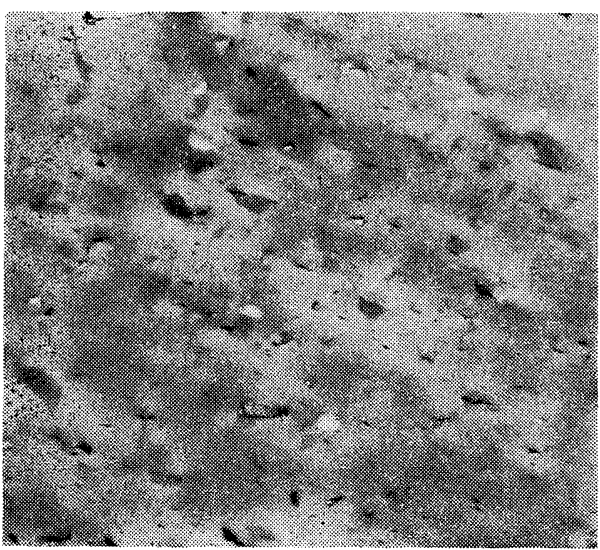

Figure 11. Electron micrograph $(\times 1,400)$ of the extrudate cross section for PVC/ABS blends (PVC/ $\mathrm{ABS}=85 / 15)$; stained and hardened by the osmium tetroxide procedure. model by introducing a fricrional correction factor into the theoretical calculation.

(2) Better agreement between theory and experiment is found for less compatible systems, such as PP/PS system and PE/PS system. Less satisfactory agreement between theory and experiment is shown for systems containing an elastic component, such as PVC/ABS and PS/PB systems.

(3) The confused phase structure of the polyblends often causes an increase in the viscosity of the system and thus the deviation from the theoretical data.

\section{APPENDIX}

The magnitude of $n$ in eq $16^{\prime}$ can be evaluated as having an order of about 100 in the real twocomponent polyblends as follows. 
The microscopic phase structure of an ideal two-component polyblend consists of a dispersed phase and a matrix base (see Figure A1(a)). When the dispersed spheres of one component is small enough in the matrix component, the number of spheres becomes equal to $\pi R^{2} L \omega_{\Lambda} /(4 / 3) \pi\left(10^{-4}\right){ }^{3}$ if the diameter of dispersed spheres is assumed to be about $1 \mu=10^{-4} \mathrm{~cm}$, and the total interface area becomes equal to $2 \pi R^{2} L \omega_{A}\left(10^{4}\right)$. On the other hand, the total contact area between two-unlike components in the proposed concentric model (see Figure A1(b)) is calculated as follows:

Consider the sum of $R_{j}$ over $j$ : This may be split into two parts: the terms with $j=2 l$ (even) and those with $j=2 l-1$ (odd).

$$
\begin{aligned}
& 2 \pi L \sum_{j=1}^{n-1} R_{j}=2 \pi L\left(\sum R_{j=2 l}+\sum R_{j=2 l-1}\right) \\
& =2 \pi L\left(\sum_{1}^{(n / 2)-1} R_{2 l}+\sum_{1}^{n / 2} R_{2 l-1}\right) \\
& =2 \pi L\left(\frac{R}{\sqrt{n}} \sum_{1}^{(n / 2)-1} \sqrt{ } l(l+1)\right. \\
& \left.+\frac{R}{\sqrt{ } n} \sum_{1}^{n / 2} \sqrt{(l-1) l+2 \omega_{\mathrm{A}} l}\right) \\
& \fallingdotseq \frac{2 \pi R L}{\sqrt{ } n}\left(\int_{1}^{(n / 2)-1} \sqrt{ } x(x+1) \mathrm{d} x\right. \\
& \left.+\int_{1}^{n / 2} \sqrt{(x-1) x+2 \omega_{\mathrm{A}} x} \mathrm{~d} x\right) \\
& \fallingdotseq \frac{2 \pi R L}{\sqrt{n}}\left(\begin{array}{c}
n^{2}(1+2 \sqrt{2}) \\
8
\end{array}\right) \text { when } n \gg 1 \\
& \fallingdotseq \pi R L n^{3 / 2}
\end{aligned}
$$

Let

$$
3 \pi R^{2} L \omega_{A}\left(10^{4}\right)=\pi R L n^{3 / 2}
$$

then

$$
n^{3 / 2}=3 R \omega_{A}\left(10^{4}\right)
$$

or

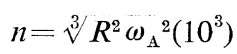

Let $R=0.1[\mathrm{~cm}]$ and $\omega_{\mathrm{A}}=0.1$, then $n$ is close to the order of about hundred.

\section{NOMENCLATURE}

$D$, Diameter across the tube, $\mathrm{cm}$; shear rate, $\mathrm{s}^{-1}$.

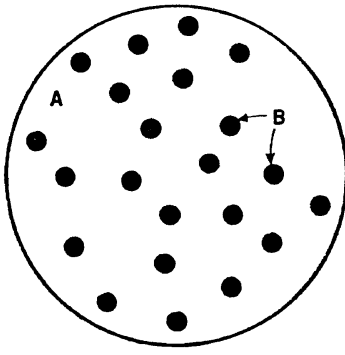

(a)

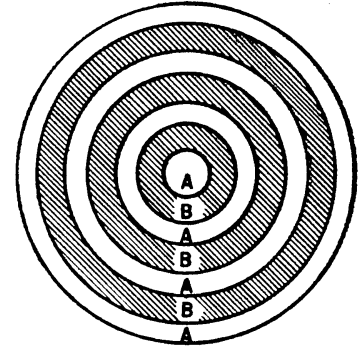

(b)
Figure A1. Comparison of particle-matrix model and concentric model for two-component polyblend.

$L$, Length of the tube, $\mathrm{cm}$.

$n$, Total number of layers in concentric adjacent fluids.

$\Delta P$, Pressure drop along the tube, $\mathrm{dyn} / \mathrm{cm}^{2}$.

$Q$, Volume flow rate of fluids, $\mathrm{cm}^{3} / \mathrm{s}$.

$R$, Radius across the tube, $\mathrm{cm}$.

$r$, Arbitrary distance from the center of tube, $\mathrm{cm}$.

$v$, Velocity profile in $i$ th layer, $\mathrm{cm} / \mathrm{s}$.

$V_{i}, \quad$ Velocity in the interface $r=R_{i}, \mathrm{~cm} / \mathrm{s}$.

$X, \quad$ Interaction of a function of $r$ and $\omega$ between the interfaces of concentric adjacent fluids, $\mathrm{dyn} / \mathrm{cm}^{2}$.

$Z$, Interaction of a function of $\omega$ between the interfaces of concentric adjacent fluids, $\mathrm{dyn} / \mathrm{cm}^{2}$.

$\alpha, \quad$ Interaction coefficient, $\mathrm{dyn} / \mathrm{cm}^{2}$.

$\beta$, Interaction parameter.

$\mu, \quad$ Viscosity of fluids, poise.

$\tau$, Shearing stress, dyn $/ \mathrm{cm}^{2}$.

$\omega$, Weight fraction.

\section{REFERENCES}

1. K. Dimov, E. Dilova, and S. Stoyanov, J. Appl. Polym. Sci. 19, 2087 (1975).

2. C. D. Han and T. C. Yu, J. Appl. Polym. Sci., 15, 1163, 2579 (1971).

3. C. D. Han and T. C. Yu, Polym. Eng. Sci., 12, 81 (1972).

4. C. D. Han, J. Appl. Polym. Sci., 18, 481 (1974).

5. R. F. Heitmiller, R. Z. Naar, and H. H. Zabusky, J. Appl. Polym. Sci., 8, 873 (1964).

6. G.N. Avgevopoulos, F.C. Weissert, P.H. Biddison, and G. G. A. Bohm, Rubber Chem. Technol., 49, 93 (1976). 